Cahiers d'études italiennes

9| 2009

NOVECENTO... E DINTORNI

Images littéraires de la société contemporaine (4)

\title{
Erri De Luca, en mal de la foi
}

\section{Nicolas Bonnet}

\section{OpenEdition}

\section{Journals}

Édition électronique

URL : http://journals.openedition.org/cei/200

DOI : $10.4000 /$ cei.200

ISSN : 2260-779X

Éditeur

UGA Éditions/Université Grenoble Alpes

Édition imprimée

Date de publication : 15 juillet 2009

Pagination : 133-144

ISBN : 978-2-84310-145-8

ISSN : $1770-9571$

Référence électronique

Nicolas Bonnet, «Erri De Luca, en mal de la foi », Cahiers d'études italiennes [En ligne], 9 | 2009, mis en ligne le 15 janvier 2011, consulté le 27 mars 2021. URL : http://journals.openedition.org/cei/200 ; DOI https://doi.org/10.4000/cei.200 


\title{
ERRI DE LUCA, EN MAL DE LA FOI
}

\author{
Nicolas Bonnet
}

Université de Bourgogne

Erri De Luca, cette figure atypique dans le panorama de la littérature italienne contemporaine, entretient avec le judaïsme et le christianisme des rapports singuliers et paradoxaux. Pour quelles raisons cet ancien militant d'extrême gauche, qui n'a pas abjuré sa foi dans les idéaux révolutionnaires de sa jeunesse et continue à se définir comme incroyant, a-t-il appris l'hébreu en autodidacte et pratique-t-il l'herméneutique biblique depuis une vingtaine d'années?

On peut ici risquer l'hypothèse que l'adhésion à Lotta Continua et la vocation d'exégète ont une même origine : la culpabilité. Aux yeux de De Luca, le manquement de ceux qui, contemporains de la nazification de l'Europe, n'ont opposé aucune résistance à la barbarie est proprement inexpiable. La faute historique des pères retombe sur leur descendance $\left(A A\right.$, p. $32 ; A$, p. $52 ; O P$, p. II9; $T M$, p. I08; $A P D R$, p. 23) ${ }^{\text {I }}$ C'est le poids de cette faute héréditaire qui pousse le jeune De Luca à épouser la cause d'une gauche extraparlementaire qui prétend dans les années 1970 renouer avec l'esprit de la Résistance pour abattre un État démocrate-chrétien qu'elle considère comme substantiellement fasciste ; et c'est la persistance de ce sentiment de culpabilité qui le pousse, après l'épuisement du mouvement révolutionnaire et sa problématique issue, à participer à différentes missions humanitaires dans le tiers-monde ou dans l'ex-Yougoslavie. C'est encore et surtout la culpabilité liée à la mémoire de la Shoah qui explique l'identification empathique de De Luca à la condition de la victime par antonomase et sa vocation sui generis de commentateur de la Thora et du Talmud.

I. Dans le commentaire de sa traduction de l'Exode, De Luca écrit : "I figli sono tenuti a disdire l'eredità paterna di una colpa, cancellandola dal proprio agire come dal patrimonio genetico. La colpa dei padri ha facilità di ricadere sui figli per imitazione, per predisposizione a ereditarla come una malattia. Ai figli incombe correggere in loro l'eventuale lascito negativo paterno. " (E/N, XXXIV, 7, note 433, p. I43) 
L'engagement politique, l'action humanitaire et l'apprentissage de l'hébreu et du Yddish relèvent de la même logique réparatrice. Il s'agit dans tous les cas de « corriger» l'irrémédiable².

De Luca s'efforce dans ses premiers travaux d'exégète d'oublier à travers une ascèse radicale le logos hellénique, de se tenir "loin d'Athènes ", matrice de la métaphysique occidentale (UNCT, p. 77-8I), et de se mettre à l'écoute d'une voix "première » irréductible aux dogmes élaborés par les religions. On relève toutefois dans le parcours exégétique de l'écrivain un glissement progressif de l'Ancien au Nouveau Testament. Si la référence aux Évangiles est pratiquement absente de ses premiers écrits, elle devient majoritaire dans ses dernières publications. In nome della Madre, tout entier centré sur le thème de la virginité mariale est un texte dogmatiquement irréprochable du point de vue de la théologie catholique et qui semble d'une inspiration quasi claudélienne dans ses meilleurs passages.

Comment peut-on rendre raison de ce passage de l'Ancienne à la Nouvelle Alliance ou plutôt de ce retour, de cette " reconversion " au catholicisme ? Bien qu'il ne s'en explique nulle part, on peut supposer qu'il s'agissait pour De Luca, dans un premier temps, de prendre ses distances par rapport à une Église qui s'était rendue complice - ne fût-ce que par son silence - du génocide. La dernière production correspond au contraire au moment de réconciliation de De Luca avec « la religion de ses pères ", et peut-être exprime-t-elle le souci de l'auteur de se réinscrire dans la filiation spirituelle qu'il avait reniée.

\section{Croyance et incroyance}

De Luca souligne que le terme " croyant " dérive d'un participe présent, que le croyant renouvelle sans cesse son credo (OP, p. 7). La foi est sujette à des variations de degrés, elle se mesure à une augmentation de température qui peut aller jusqu'à l'embrasement de l'être ( $A$, p. II8). Ce n'est pas

2. On peut se demander si la dette héréditaire, liée à une faute historiquement déterminée, ne masque pas, dans l'imaginaire deluchien, une culpabilité plus radicale, proprement originaire. Le narrateur du premier roman de De Luca, Non ora, non qui, raconte qu'enfant, à force d'écouter sa mère lui faire part des malheurs qui accablent le monde, il s'était persuadé, dans un délire paranoïaque d'inspiration vaguement gnostique, qu'il n'était autre que le Verbe incarné, le Fils abandonné sur cette terre par le Père impuissant, et comptable du mal inhérent à la création : "Allora, non so proprio come fu, io capii che non ero testimone di tutto quel male e del mondo, ma responsabile. Tu lo enumeravi e me ne chiedevi conto solo nominandolo. Si mamma, sotto il silenzio assorto un bambino credette di essere l'ultimo pezzo di Dio, frammento scollato d'un creatore al quale l'opera era sfuggita di bocca e di mano. Non sapeva più che fare o che dire, il Dio in quel bambino, tranne ascoltare. " (NONQ, p. 58-59) 
un état stable et l'homme de foi passe nécessairement par toutes les « positions » allant du sentiment de déréliction à " la puissante certitude que Dieu est près de soi » $(A, \mathrm{p}$. II 5$)$. De Luca se définit négativement comme "quelqu'un qui ne croit pas ». C'est un agnostique qui refuse la dénomination d'athée $(O P, \mathrm{p} .7)$. "J'attache de la valeur à l'usage du verbe aimer et à l'hypothèse qu'il existe un créateur " soutient le poète (OSA, p. 98). Loin de professer comme Gianni Vattimo la foi " restreinte » du demi-croyant (Vattimo, $C D C$, p. 77), il se proclame sans ambiguïté « incroyant " mais se dit aussi mû par "l'aiguillon du manque » et l'insatiable soif de sens que nulle explication ne saurait combler ( $A$, p. IO4). On pourrait parler dans son cas, en renversant la proposition de Saint Anselme, d'un intellectus quaerens fidem, d'une raison en quête de la foi. De Luca se défie de la rationalité et fustige la crédulité de ceux qui, cessant de croire " en de grandes choses ", versent dans les formes modernes de l'idolâtrie et de la superstition que favorise notre civilisation sécularisée et techniciste $(A$, p. 5I). Étranger à la néo-scolastique autant qu'au modernisme chrétien, il refuse toute réponse rationnelle à la question ontologique, toute tentative d'explication scientifique de la genèse, fût-elle en accord avec l'enseignement des Écritures: "Sono poco sensibile ai punti di contatto tra la scienza e la scrittura sacra. Quella storia è più grande di una sua dimostrazione scientifica. Non è riducibile a verifica" $(A, \mathrm{p}$. I6). De Luca se rattache à la tradition fidéiste : la foi, à ses yeux, est « sans preuves » :

Non riesco a partecipare del fervore di disputa intorno a un frammento che daterebbe un po' all'indietro il vangelo di Marco o il tessuto della sindone. Le notizie delle sacre scritture obbediscono a un'altra legge di verifica: se hai fede o no. Esse contengono articoli non addomesticabili a prove. Prendere o lasciare, ma non secondo le ultime perizie medico-legali. Se invece si cercano segni, allora la parola è a Marco (8, Io). "Perché questa generazione chiede un segno? Vi dico in verità che nessun segno sarà dato a questa generazione." ( $A, \mathrm{p} .76)$

Bien qu'il n'emploie pas le terme de grâce, De Luca définit la foi comme un don de l'Esprit reçu indépendamment de toute initiative du sujet :

La fede non è una cosa automatica, la fede è come quella specie di pala al vento che ho montato quando sono stato in Africa. Uno strumento molto semplice, molto rudimentale, che serve a tirare fuori l'acqua dai pozzi; però anche molto potente, che non ha bisogno di nessun tipo di manutenzione: non è necessario azionarla, va da sola, ha bisogno solo di vento. Ecco la fede è così, chi la prova è perché è stato messo in moto tutto insieme da questo impatto col vento. ( $A P D R$, p. 56$)$ 


\section{Obstacles et médiations}

Dans la préface à Noccciolo d'oliva, De Luca fait état de deux obstacles qu'il rencontre dans son cheminement vers la foi. Le premier tient à son incapacité à pratiquer la prière. Il se définit comme "quelqu'un qui ne sait pas s'adresser à Dieu " (NDO, p. 6), qui ne parvient pas à l'interpeller, fût-ce pour récriminer ou blasphémer, comme le fait Job (OP, p. 9-II). Seul le croyant est capable de ce tutoiement qui réduit, sans l'abolir, la distance entre le Créateur et sa créature ( $A$, p. 36) :

L'ultima volta che quel tu mi ha fatto sobbalzare fu mentre accompagnavo, con una piccola folla, alla sepoltura una bambina di dieci anni morta di cancro. Nel fruscio dei passi del piccolo corteo si levò d'improvviso il grido terribile del padre di quell'unica figlia, un muratore mio compagno di lavoro. Gridò: "Torturatore, me l'hai fatta torturare per un anno, mi fai schifo", gridò dritto al cielo le sue bestemmie guardando in alto e poi sputando in terra, lui ateo di sempre. Era il "tu" di un uomo a Dio, un tu antico che veniva dagli urli dei profeti e dopo un lungo sonno s'impennava nelle mie orecchie in un fiato di puro dolore. Quel "tu" era così forte che dimostrava l'esistenza di Dio almeno in quell'ora e in quell'uomo. (OP, p. 79-80)

Par la force de l'apostrophe, par la vertu performative de la prière, le sujet fait advenir Dieu. C'est aussi ce qu'affirme Rav Daniel dans Montedidio : "Rafaniello dice che a forza di insistere Dio è costretto a esistere, a forza di preghiere si forma il suo orecchio, a forza di lacrime nostre i suoi occhi vedono, a forza di allegria spunta il suo sorriso " $(M$, p. 57). À défaut de pouvoir parler «directement " à Dieu ${ }^{3}$, De Luca parle de Dieu ${ }^{4}$ ce qui place tout son discours sous le signe de "la séparation " radicale $(O P$, p. 8-IO ; NDO, p. 5-6). Le deuxième obstacle est le pardon :

L'altro inciampo è il perdono. Non so perdonare e non posso ammettere di essere perdonato. È bestemmia per il credente, per lui non c’è colpa che non possa essere sollevata da Dio. [...] Nella mia vita c'è una soglia dell'imperdonabile. Non posso ammettere di essere perdonato, non so perdonare quello che è commesso. Ecco le mie pietre di inciampo per le quali resto fuori dalla comunità dei credenti. (OP, p. 7-8)

3. Ce n'est que par la médiation de la fiction que De Luca peut adopter le langage de la prière. Le narrateur de Nave di Esilio, imagine qu'il fait parti des rescapés de la Shoah, de ces survivants à la fois " reste " et "principe ", "rebut " et " début " d'un nouveau peuple ( $A$, p. 96), et qu’il s'est embarqué, "voyageur illégal ", parmi les passagers de l'Exodus à destination de la Palestine. Il adresse à Dieu la prière Écoute Israël qu'il paraphrase et commente en assumant dramatiquement la première personne chorale, le " nous " de la communauté juive (OP, p. II9-I25).

4. Le récit à la troisième personne n'est pas la seule modalité narrative qu'exploite De Luca : dans l'un de ses textes, l'auteur qui par ailleurs reproche au Thomas Mann de Joseph en Égypte d'avoir usurpé la place du Créateur et " parodié » la Bible $(A$, p. 66) n'hésite pas à prêter sa voix au Verbe Incarné, à raconter à la première personne sa Passion et sa Résurrection dans Dal fresco di una cantina di un sepolcro (NDO, p. 27-3I). 
Ce deuxième obstacle est plus inattendu. À la différence de la prière qui n'a de sens que dans l'ordre du religieux, le pardon est aussi une action éthique et pourrait parfaitement se concevoir dans une perspective " horizontale ", purement laïque, hors de toute référence à la transcendance. Mais pour De Luca, proche sur ce point de la tradition janséniste, sans la médiation de celui " qui enlève les péchés du monde », il ne saurait y avoir de véritable rémission C'est une position de principe d'ordre métaphysique qui ne prend en considération ni l'attitude de l'offenseur (son éventuelle repentance) ni celle de l'offensé (sa liberté de concéder le pardon). Aux antipodes de Derrida qui voit dans le pardon "impossible ", dans le pardon de l'impardonnable, le seul acte conforme à son concept en tant que « perfection du don " (Derrida, FES, p. IOI-I33), De Luca considère ainsi toute faute comme irrémissible (NDO, p. $8 ; I C D U$, p. 59-6I). Son pessimisme fait écho sur ce point à celui de l'Ecclésiastes. Telle est la condition d'une créature privée de la grâce : misère pascalienne de l'homme sans Dieu.

À défaut d'avoir reçu la grâce, De Luca a foi par procuration, il a foi en la foi d'autrui : "Io non sono stato toccato da questo vento però la vita di certi uomini di fede mi convince, credo che loro abbiano una ragione, una notizia, una verità che io non ho. Credo alle vite, alle loro vite" (APDR, p. 56 ; voir aussi $P$, p. 52). Celui qui n'a pas la foi peut au moins reconnaître dans celui qui en est animé « l'empreinte digitale, la trace de la chaussure de Dieu » et, devenant par là " témoin indirect ", annoncer qu'il a vu « dans un autre la nouvelle» (IAAS, p. I24; NDO, p. 39).

\section{Vocation}

De Luca se montre proche de l'esprit de la réforme en ce qu'il invite à un dialogue direct avec les Écritures, mais il n'est certainement pas luthérien au sens où il considérerait que le salut dépend de la "seule foi ». Il ne cesse en effet de revenir dans ses essais sur la nécessité des œuvres. L'absence de vocation qui affecte les nouvelles générations est profondément mortifère. Les désespérés et les suicidaires d'aujourd'hui seraient, à l'opposé des premiers chrétiens qui aspiraient au sacrifice et au martyre afin de témoigner

5. En note de sa traduction du $15^{\mathrm{e}}$ verset du livre I de l'Ecclésiaste (Un torto fatto non potrà raddrizzarsi. E ciò che manca non potrà essere contato) De Luca commente : " [...] un' ingiustizia commessa non si può pesare, né misurare. Nessun risarcimento riscatta il male fatto. Ė distanza abissale dal pentimento e dall'assoluzione. $K$. Sa che il torto è irrevocabile" (K/E, I, I5, note 23, p. 25). Le narrateur de Non ora, non qui affirme : "Il male è irreparabile e non c'è modo di risanare un torto qualunque cosa si faccia dopo. Non c'è rimedio al di fuori di non commetterli e non commetterli è opera la più ardua e segreta in mezzo al mondo." (NONQ, p. 26) 
de leur foi, les «oubliés d'un appel " $(A, \mathrm{p} \text {. I2I })^{6}$. De Luca souligne le caractère paradoxal de l'élection. Les Écritures sont pleines d'individus requis par le Seigneur contre leur gré et sommés par lui d'accomplir une mission à laquelle ils ne sont nullement préparés (ibidem, p. 40); tous ces appelés "sans qualification " sont des figures "donquichottesques " ante litteram $^{7}$. Bien qu'il affirme ne pas avoir lui-même la vocation, De Luca marche sur les pas de ceux qui ont répondu à l'appel. C'est en ces termes qu'il évoque sa participation aux missions humanitaires aux côtés des volontaires de la Caritas: "Vado con loro perché da solo non avrei mai trovato o nemmeno cercato la pace, la pista per metterci i passi. Credo che quelli come loro hanno eseguito l'ordine del comandante Davide: "Cerca pace e inseguila" " $(A$, p. 64 ; voir aussi p. 72). En bon théologien, De Luca refuse de réduire la charité à une simple éthique. Il sait que si l'acte n'est pas porté par la foi, transcendé par la grâce, il reste inachevé, privée de sa dimension eschatologique :

Gli amici della carovana pregano il loro rosario ogni giorno, ascoltano messa, salgono sulla collina delle apparizioni. Calcano cose sacre nei passi e nella voce. Sento la differenza da loro in questo strano spessore che i miei gesti non hanno. Il mio scaricare casse è solo quello, non porta altro, il loro scaricare casse è invece come un coccio di vetro che da terra rifrange luce in tutte le direzioni, ma soprattutto in cielo. Hanno da dire questo: che le opere, la buona volontà è ancora niente, un'ombra appena, mentre a me paiono tutto e sono venuto con loro solo per quelle cose. Perciò intendo a mio modo, poco, che i loro gesti durano ei miei no. Sono solo uno che legge la Bibbia, loro sono quelli che la reggono. ( $P$, p. 93-94)

Le bibliste incrédule ne se satisfait pas d'un évangile sécularisé et demeure dans l'attente de la grâce.

\section{Sacré}

Conscient que le religieux est réapparu sous sa forme la plus sectaire et la plus intolérante, et que les hommes se font la guerre " au nom de Dieu » ( $A$, p. 6I), De Luca distingue nettement la foi du fanatisme et fustige la prétention de chaque communauté à être seule détentrice de la vraie foi :

6. Les suicidés semblent s'être chargés de tout le mal du monde, pour en alléger autrui, et avoir succombé sous son poids (ibidem, p. 47).

7. De Luca propose dans Chisciottimista une interprétation à rebours du Quichotte (très proche de la lecture " aberrante " d'Unamuno auquel il ne se réfere toutefois jamais et qu'il ignore peut-être), transformant l'extravagant héros de Cervantès en une figure messianique ( $C$, p. 8-Io). 
Avanza con cenni sicuri un secolo che viene a innalzare armi e versetti. Prima che nuovi agitatori del sacro vengano con la Bibbia in pugno ad arrostire genti sforzando le sue pagine a dettare massacri, racconto quello che ho trovato leggendo quel libro nella sua lingua madre. / Presto alcuni nomi di Dio issati su opposte bandiere e i popoli saranno nemici per una volta ancora, in nome del cielo. Prima dei regni delle fedi armate, ognuno s'affretti a leggere la Bibbia a modo suo, con fervore e freddezza, in buona o cattiva sorte. (UNCT, quatrième de couverture)

Si le texte n'appartient à aucune communauté d'interprètes, l'interprète peut au contraire éprouver son appartenance au livre : c'est en ces termes que l'essayiste s'exprime dans Altre prove di risposta : "Non mi sento di appartenere ad alcuna gente e comunità, ma a quel libro sì, a quello appartengo" (APDR, p. 59). Toutefois, dans Nocciolo d'oliva, De Luca ne se définit plus comme un "résident » mais comme un simple " passant » des Écritures (NDO, p. 39) et dans Napòlide, il va jusqu'à renverser sa première assertion : "Non ho cercato asilo in quella lingua, né appartenenza. [...] Arrivavo all'ebraico delle Scritture per bisogno di starmene lontano" $(N$, p. 22). Bien qu'il prétende rester au seuil de la Révélation, il s'exprime souvent dans ses livres comme s'il l'habitait - en était habité -, sa posture improbable est celle du sceptique qui s'identifie au mystique, son langage sentencieux, aux accents prophétiques, est celui, contradictoire, d'un prêcheur agnostique.

De Luca se définit comme quelqu'un qui "n'arrive pas à la foi » et "s'arrête à la beauté des histoires qu'il lit et relit tous les jours " (OP, p. I8). Il ne doute pas toutefois que cette beauté soit au service d'un dessein transcendant : les Écritures, incomparables aux autres livres, ne sauraient être l'objet d'une jouissance purement esthétique : " [...] la Bibbia mi ha insegnato a correggere la vanità dei libri, collocandoli all'altezza del suolo, tra $i$ piedi, le scarpe e le scope " $(P, \mathrm{p} . \mathrm{I} 3)^{8}$. De Luca affirme "la priorité de l'écoute " (NDO, p. 40-4I) ; la réception doit s'entendre ici dans son acception la plus littérale : il s'agit de faire le vide en soi et de s'isoler de la rumeur du monde ( $A$, p. I8) pour "accueillir " la Révélation (UNCT, p. 9). Pour rendre compte de son expérience de lecteur, De Luca affirme qu'il se laisse envahir par le texte : "Ogni mattino a testa vuota e lenta accolgo le parole sacre. Capirle per me non è afferrarle, ma essere raggiunto

8. Bien qu’il ne mette pas la littérature profane sur le même plan que la Bible, De Luca, renouvelant le topos de l'artiste inspiré, assimile le poète au prophète. Celui-ci est, comme celui-là, traversé par des voix $(A$, p. 87$)$, il doit, écrit De Luca, «se laisser jouer sans la prétention d'être l'instrumentiste, le compositeur » (ibidem, p. 93). La littérature, dans cette perspective sacralisante, peut s'offrir comme un ultime recours, comme un substitut de la grâce : "Chi è messo alle strette o ha il cielo o ha i libri in tutti e due i casi la sua solitudine è invasa dalle voci più belle del mondo. " (APDR, p. 24) 
da loro, essere cosi quieto da farsi agitare da loro, cosi privo d'intenzione da ricevere la loro e cosi insipido da farsene salare » (OP, p. 6). De Luca se dit docile, soumis au texte: "Non ho adattato il testo ad una interpretazione, ne sono stato invece piegato" (UNCT, p. 9). On est aux antipodes de l'antiessentialisme et du pragmatisme radical d'un Richard Rorty qui identifie utilisation et interprétation et pour qui tout lecteur façonne le texte et produit le sens en fonction de ses fins (Rorty, COP). De Luca revendique une lecture littérale de la Bible (UNCT, p. Io), il veut témoigner de « la plus stricte obédience à la surface révélée » $(O P$, p. 8$)$, n’entendre que « la lettre nue » (ibidem, p. I3), recevoir « l'immensité du sens même en restant à la surface des mots " (NDO, p. 8). En tant que traducteur, il appartient à la famille des "sourciers ": "Provo un attaccamento molto forte a quella lingua al punto da volerla tradurre, da voler provare a tradurla nella maniera più piatta possibile... " (APDR, p. 59). Ce souci de littéralité implique la pratique du calque syntaxique (NDO, p. 40) et l'emploi dans la langue cible d'un lexique réduit à cinq mille mots (ibidem, p. 47). On aura beau jeu de taxer De Luca de naïveté philologique, de rappeler le paradoxe du cercle herméneutique en vertu duquel toute lecture est nécessairement déjà interprétation, c'est-à-dire construction du sens selon l'intentionnalité du lecteur et l'horizon de la communauté des interprètes à laquelle il appartient (cf. E. Garroni, in Lavagetto, ITL, p. 245-282).

Le premier chapitre de Una nuvola come tappeto consacré à Babel semble apporter un démenti au littéralisme programmatique de De Luca : le commentateur réinterprète le texte, c'est-à-dire qu'il lui donne un nouveau sens en renversant, comme le fit avant lui George Steiner dans After Babel, la tradition exégétique : dans cette perspective paradoxale, la multiplication des langues n'est plus perçue comme une malédiction mais comme une bénédiction. Or, on ne voit pas que la lettre du texte autorise une telle lecture qui est loin de tomber sous le sens ${ }^{9}$. Dans le langage de David Hirsch, on pourrait dire que la signification qu'acquiert le texte à l'occasion de sa nouvelle réception ne coïncide pas avec son sens philologique (Hirsch, p. vir). Toutefois, la lecture littérale, telle que l'entend De Luca, n'est pas une lecture univoque et définitive : le texte doit s'entendre, selon l'expression rimbaldienne, "à la lettre et dans tous les sens ". C'est donc une lecture qui n'a rien de "fondamentaliste " mais se veut « ouverte ». Le lecteur se retrouve dans le texte qui s'adresse à lui :

9. En dépit de la subtile mais peu probante analyse lexicale exposée dans Voce del verbo scendere in E. De Luca et G. Matino, Sottosopra, p. 4I. 
Leggere i libri sacri dà a volte la sorpresa di trovare se stessi in certi versi. Allora ci si sente raggiunti come d'estate dal frammento di cometa che s'incendia proprio davanti ai nostri occhi spalancati al buio / [... Perché il libro, anche il sacro, appartiene a chi lo legge [...]. Perché ogni lettore pretende che in un rotolo di libro ci sia qualcosa scritto su di lui. ( $A$, p. II7)

De Luca affirme à propos de sa pratique du texte : "questa frequentazione è tutta l'autorità di cui dispongo" (UNCT, p. I2). On est proche, à première vue, de la position calviniste qui ne reconnaît d'autorité qu'au Texte et exhorte les fidèles à une pratique directe du Livre en rejetant la médiation de l'Église et en disqualifiant la communauté des docteurs dépositaires du sens et garants de l'interprétation. Toutefois, De Luca souligne qu'au caractère sacré que présente en soi la Bible «s'ajoute » celui que lui confère la tradition herméneutique millénaire dont elle est l'objet :

Per molti la Bibbia è un testo sacro. Ma mi commuove più di quel valore in sé, il sacro aggiunto, l'opera degli innumerevoli lettori, commentatori, sapienti che hanno dedicato a quel libro il tempo migliore della loro vita. Il sacro in sé della Bibbia è diventato attraverso di loro una civiltà. (UNCT, p. 9-Io)

De Luca prétend « suivre le sillon » tracé par la tradition en « ajoutant quelque chose » que le texte " contenait " mais qui «n'avait pas encore été exprimé » (ibidem, p. Io) : le sens découvert par l'interprète est bien à ses yeux une propriété de l'œuvre. Chacun est ainsi susceptible d'apporter sa contribution en actualisant un sens virtuellement présent dans le texte. Non seulement le travail herméneutique est un processus ad infinitum mais l'interprétation ignore le principe de non-contradiction et n'est soumise à aucune procédure de validation, bien que les références aux autorités rabbiniques, véritables garde-fous, abondent dans l'exégèse deluchienne de la Thora (NDO, p. 50, 80 et 88).

Il Talmud è pieno di interpretazioni diverse di uno stesso verso e nessuna annulla l'altra. Perché la rivelazione non si interrompe mai, offre scoperte nuove anche all'ultimo dei suoi lettori. Anche lui può aggiungere il suo lume al verso che gli è toccato in sorte d'incontrare. È la sua eredità e lui è l'eredità di quel verso. Aggiunge il suo commento all'infinita stesura non con la pretesa di aumentare conoscenza, ma testimoniare della propria riconoscenza. $(A$, p. 27$)$

Même « le dernier venu " peut grappiller la vigne et trouver son bonheur après les vendanges ( $O P$, p. I9-2I ; NDO, p. II9-I2I). La gratitude du lecteur naif s'oppose à l'orgueil du spécialiste qui se croit maître du sens, oubliant que le texte n'est la propriété de personne ( $A$, p. 90).

Alors que Gianni Vattimo se pense fidèle à l'enseignement du Christ en rejetant les prescriptions bibliques qu'il estime en contradiction avec 
" le commandement suprême de la charité ", noyau irréductible à ses yeux du message évangélique (Vattimo, $C D C$, p. 76), De Luca refuse d'exercer un tel inventaire. Son attitude à l'égard des préceptes des Écritures est aux antipodes de l'approche déconstructionniste pratiquée par Derrida et ses disciples s'attaquant au "phallogocentrisme " biblique. S’il est vrai qu'il évoque «la maternité » de Dieu, proposition audacieuse qui implique que l'on s'écarte pour une fois de la lettre, c'est en accord avec la théologie de Vatican II (A, p. 68-69). Afin de trouver une justification au fait que la deuxième personne est au masculin dans le texte du décalogue, il risque l'hypothèse que le choix du genre tient à la différence des rôles impartis aux deux sexes par le créateur : il appartiendrait à la femme de transmettre la vie et à l'homme la loi : "Questa è solo una mia ipotesi di spiegazione circa l'uso del tu maschile nel decalogo. La nomino insieme a un tempo in cui i sessi non giocavano a scambiarsi i vestiti" ( $A, \mathrm{p} .28)$. L'expression : "un temps où les sexes ne jouaient pas à s'échanger leurs vêtements " révèle que de Luca n'envisage pas la conception judéo-chrétienne des identités sexuelles dans la perspective historique de l'anthropologie culturelle mais comme un absolu : "Nella scrittura sacra $i$ sessi sono ben separati e la loro unione è la più forte alleanza tra creature " (NDO, p. 53). De tels exemples illustrent la limite du commentaire deluchien - limite parfaitement assumée - : le refus de tout rapport critique au texte ; celui-ci, "irréductible à l'œuvre d'auteurs variés " (NDO, p. 7), n'est interrogé qu'en tant qu'il est supposé délivrer une vérité universelle : "Alcune pagine, alcune parole mi hanno rivelato qualcosa della loro verità e mi hanno istigato a darne notizia" $\left(U N C T\right.$, p. 9) ${ }^{\text {Io }}$. Il n'est jusqu'au casse-tête philologique imputable à une probable corruption du texte qui ne recèle quelque « intention sacrée » :

Credo che anche i pezzi gualciti, ammaccati, facciano parte della provvidenza di quelle scritture. Anche l'errore, se di esso si tratta, custodisce un'intenzione sacra. Nella mia lettura, quando non capisco, lascio in bianco. Attribuisco volentieri l'incomprensione alla mia debolezza d'intendere. Infine credo che tutte le parole zoppe delle scritture siano lì per apparecchiare il riso di Iod/ Dio, l'autore. Attraverso dei copisti un poco ciechi e sordi ha fatto deragliare qualche parola: quando le sente pronunciare, ride. Perciò non dobbiamo correggerle, inseguire la probabile esattezza. Dobbiamo far ridere Dio: ci riusciamo solo noi. $(A$, p. 105$)$

La tradition herméneutique est une perpétuelle dérive et le retour aux sources s'avère proprement impossible. Le narrateur d'Aceto, Arcobaleno

Io. On mesure à ce propos l'ambiguïté d'une démarche qui consiste à mettre son propre verbe au service d'une révélation à laquelle on prétend ne pas adhérer soi-même, à se faire le porte-voix de la nouvelle sans y prêter personnellement foi. 
ne présente pas la transmission en termes d'accroissement mais au contraire de graduelle et inéluctable exténuation du sens :

Molte volte ho pensato con nostalgia che le generazioni si sono trasmesse questo medesimo libro leggendo in esso sempre qualcosa in meno, proprio come se un unico dito, scorrendo una sola copia, lentamente ne cancellasse le righe. Essere molto sfiorato: la somma di molte carezze è un'abrasione. Ma prima che essa si compia sorge una generazione di fedeltà che prova a ricalcare quelle preziose lettere consunte. In molti punti dovrà ricostruirle con immaginazione. La più intensa lealtà, osando il restauro dell'irreparabile, coincide con la contraffazione. Stanotte questo pensiero non mi intristisce, non temo più le rovine dell'originale: ora vi riconosco l'opera di una provvidenza che attraverso la nostra cecità riscrive il suo libro. Così la perdita ha un vincolo di scambio con l'acquisto, le parole scomparse riaffiorano altrove. ( $A A$, p. 72)

Le sens originaire du Texte est définitivement perdu et la tentative de restauration philologique est une illusion créatrice. Toutefois, De Luca (ou du moins le narrateur du roman) n'est pas gadamérien : bien que la validité de l'interprétation s'inscrive dans l'horizon d'une époque, elle ne saurait à ses yeux se fonder exclusivement sur le consensus de la communauté des interprètes. La réinterprétation du legs de la tradition en fonction du nouvel horizon n'est pas un processus historique immanent car «la réécriture du Livre » est « l'œuvre de la providence ». C'est pourquoi la réactualisation n'est pas une trahison de l'intention originaire mais participe au contraire du déploiement de la révélation : l'interprète s'exprime toujours sous le contrôle de l'auteur.

\section{Bibliographie}

De Luca E., Non ora, non qui [1989], Milano, Feltrinelli, coll. « Universale Economica ", 1992.

—, Una nuvola come tappeto [1991], Milano, Feltrinelli, coll. «Universale Economica ", 1994.

—, Aceto, Arcobaleno [1992], Milano, Feltrinelli, coll. « Universale Economica ", 1992.

—, In alto a sinistra [1994], Milano, Feltrinelli, coll. « Universale Economica ", I995.

—, Esodo / Nomi (traduction commentée), Milano, Feltrinelli, coll. «Universale Economica - I Classici ", I994.

—, Pianoterra, Macerata, Quodlibet, 1995. 
—, Kohèlet / Ecclesiaste [1996] (traduction commentée), Milano, Feltrinelli, coll. « Universale Economica - I Classici », 1996.

—, Alzaia [1997], Milano, Feltrinelli, coll. «Universale Economica Super UE » (édition augmentée), 2004.

—, Ora prima, Magnano (BI), Edizioni Qiqajon Comunità di Bose, 1997.

—, Tu, Mio [1998], Milano, Feltrinelli, coll. "Universale Economica », 2000.

—, Altre prove di risposta, Napoli, Libreria Dante \& Descartes, 2000.

—, Montedidio [200I], Milano, Feltrinelli, coll. «Universale Economica », 2003.

—, Nocciolo d'oliva, Padova, Edizioni Messaggero, 2002.

—, Opere sull'acqua (édition bilingue, trad. Danièle Valin), Paris, Seghers, 2002.

-, Il contrario di uno [2003], Milano, Feltrinelli, 2005.

-, Solo andata, Milano, Feltrinelli, 2005.

—, Chisciottimista, Napoli, Libreria Dante \& Descartes, 2005.

—, In nome della madre, Milano, Feltrinelli, 2006.

—, Napòlide, Napoli, Libreria Dante \& Descartes, 2006.

De Luca E. e Matino G., Sottosopra, Alture dell'antico e del nuovo testamento, Milano, Mondadori, 2007.

Derrida J., Foi et savoir. Le siècle et le pardon, Paris, Seuil, "Points ", 2000.

Hirsch D. E., Validity in Interpretation, New Haven and London, Yale University Press, 1967.

Lavagetto M. (éd.), Il testo letterario, Istruzione per l'uso [1996], Bari, Laterza, 200I.

Rorty R., Consequences of pragmatism, Minneapolis, University of Minnesota Press, 1982.

Steiner G., After Babel. Aspects of Language and Translation, Oxford, University Press, 1977.

Vatтimo G., Credere di credere, È possibile essere cristiani nonostante la chiesa?, Milano, Garzanti, 1998. 